\title{
In Silico Analysis of 716 Natural Bioactive Molecules Form Atlantic Ocean Reveals Candidate Molecule to Inhibit Spike Protein
}

Peter T. Habib ( $\sim$ p.habib911@gmail.com )

Colors Medical Laboratories

Maha Saber-Ayad

University of Sharjah

Sameh E. Hassanein

Agricultural Research Center (ARC)

\section{Research Article}

Keywords: COVID-19, Spike, Medicinal Marine Compounds, Molecular Docking, Virtual Screening

Posted Date: February 4th, 2021

DOl: https://doi.org/10.21203/rs.3.rs-156623/v1

License: (1) This work is licensed under a Creative Commons Attribution 4.0 International License.

Read Full License 


\section{Abstract}

COVID-19, a new pandemic of coronavirus (CoV), was reported in Wuhan, China, in 2019. No specific drugs are available and investigations regarding COVID-19 treatment are proceeding. Lan et al. (2020) successfully crystallized the COVID-19 spike receptor-binding domain bounding to the ACE2 receptor, which is a potential drug target. The present study aimed to assess 716 bioactive compounds found in the South Atlantic Ocean as potential COVID-19 Spike inhibitors, using a molecular docking study. Molecular docking was performed using Autodock Vina to analyze the probability of docking. COVID-19 Spike was docked with several compounds, and docking was virtually screened by Chimera, Pymol, and Biovia Discovery Studio and test for draggability using SWISSADME. The analysis shows that 11 NPs out of 716 are predicted to be Spike inhibitors by blocking the amino acids responsible for binding Spike to ACE2. However, further findings are necessary to experimentally investigated for their potential medicinal use.

\section{Introduction}

A global health catastrophe has been created by SARS CoV-2 (COVID-19) This pandemic-threatening situation closely resembles the 2003 SARS CoV outbreak (Walls et al. 2020). But with an extremely higher degree of virulence, the current one is highly spreading. As of today, it has killed over 2,169,344 thousand people in 210 countries out of a total of 100,913,073 infected people until the time of this paper (https://www.worldometers.info/coronavirus/). The world economy in most places of the globe has been in a jerk under 4 months or even more of a quarantine period. Thus therapeutic/preventive action is an immediate necessity and a challenging act against this highly stable and often mutable viral strain. No therapeutics are developed against this infection. A few of the old medications are used from similar types of diseases, based on previous observations. In some in vitro models, some experiments were performed with inclusive findings. For instance, some recent study suggests that in an experimental in vitro model, remdesivir and chloroquine effectively inhibit this infection (Wang et al., 2020; Cortegiani et al. 2020; Gao and Tian, 2020). But chloroquine may have high toxicity, and it is predicted that hydroxychloroquine is less toxic than it is (Colson, Rolain, and Raoult 2020). Hydroxychloroquine treatment is strongly correlated with viral load reduction in COVID-19 patients in a trial and survey-type experiment with very limited sample size, and its effect is enhanced by azithromycin (Gautret et al. 2020) . Out of a few combinations of medication, presently HCQ is being used in COVID-19 cases (Wang et al., n.d.). Apart from that, this drug is also used in systemic lupus erythematosus (SLE) and rheumatoid arthritis (RA) among other autoimmune diseases. Other drugs such as remdesivir, lopinavir/ritonavir combination, Favipiravir have demonstrated effectiveness in inhibiting coronavirus in vitro. It has been shown that teicoplanin, an antibiotic used to treat staphylococcal infections, inhibits MERS-CoV in the human cells. In the current pandemic, this medication can also be rechecked (Baron et al. 2020). Studies on molecular docking propose sofosbuvir, galidesivir, and tenofovir to inactivate SARS-CoV2 RNA Dependent RNA Polymerase (Elfiky 2020). 
In terms of its biological diversity, South Africa ranks third in the world (Griffiths et al. 2010), and it is estimated that 3,000 medicinal plant species are used for therapeutic purposes in this region, mostly as part of traditional medicines (Cragg and Newman 2013). Nearly every documented disease has been treated with natural products (NPs), with reports from civilizations all over the world going back as far as 4,600 years ago (Cragg and Newman 2013; Dias, Urban, and Roessner 2012). NPs are the natural consequence of evolutionary processes that provide them with a level of structural complexity, chemical diversity, and biological proprieties not seen in simply synthetic compounds (Pelay-Gimeno, Tulla-Puche, and Albericio 2013; Mishra and Tiwari 2011). NPs are considered essential for the drug development process (Mishra and Tiwari 2011) and about 64 percent of approved drugs were derived from or influenced by NPs between 1981-2010 (Newman and Cragg 2012). There has been one since 2008. 25 Newly authorized drugs derived from NPs, with 31 additional drugs whether in phase III clinical trials or past phase III (Butler, Robertson, and Cooper 2014).

Clarkson (Clarkson et al. 2004) reported that 49 percent of the 134 different South African medicinal plant extracts tested had at least moderate antispasmodic activity. Similar manner, Besson (Bessong et al. 2005) documented a series of South African plant extracts that showed action against HIV. van Vuuren (Van Vuuren 2008) discusses the antimicrobial activity of South African plant life, including examples of different compounds isolated from these plants and their antimicrobial activity as measured. Marine NPs are a fairly new origin of pharmaceutical agents (Glaser and Mayer 2009) but they exhibit complex and special chemistry (Haefner 2003). Despite early research efforts, Southern African aquatic chemistry work only really took off in the 1990s with isolated molecules showing great potential as anticancer agents (Davies-Coleman and Beukes 2004).

The methodology of molecular docking explores the behavior of small molecules at a target protein's binding position. With more protein structures being experimentally determined using spectroscopy of Xray crystallography or nuclear magnetic resonance (NMR), molecular docking is increasingly being used as a method in drug discovery. It also becomes possible to dock against homology-modeled targets for proteins whose structures are not known. For further lead optimization processes, the docking strategies can be used to measure the druggability of the compounds and their specificity against a given target. Molecular docking programs carry out a search algorithm in which the ligand conformation is evaluated continuously until the minimum energy state is reached. Finally, an affinity scoring method, $\Delta G$ [U total in $\mathrm{kcal} / \mathrm{mol}]$, is used to rate the candidate as the sum of the electrostatic energies and van der Waals. The driving forces in biological systems for these specific interactions aim at the complementarities between the shape and electrostatics of the binding site surfaces and the ligand or substratum.

Molecular docking shows massive potentials in discovery of several molecules have the ability inhibit Coronavirus proteins. Sharma (Sharma and Kaur 2020) suggest Jensenone to be main protease (Mpro) inhibitor. Ghosh (Ghosh et al. 2020) report significant inhibition potential of three polyphenols from green tea against Mpro. In another studies, FDA approved antiviral drug against COVID-19 shows IDX-184 is superior compared to Ribavirin (Elfiky, Mahdy, and Elshemey 2017). flavonoid from medicinal plant 
(Adem et al. 2020) and eucalyptol oil component from eucalyptus oil (Sharma and others 2020) have activity against Mpro.

In this study, we used Chimera(Pettersen et al. 2004), OpenBabel (O'Boyle et al. 2011), Pymol (DeLano and others 2002), Autodock Vina (Trott and Olson 2010), and LigPlot+ (Laskowski and Swindells 2011) to virtually screened docked 716 NPs against the binding domain of Spike protein that bound to ACE2 domain. We docked the NPs to the area in which the amino acids responsible for binding to the ACE2 receptor.

\section{Material And Methods}

\section{Spike and Ligands Download}

We collect the 716 ligand downloaded from SANCDB (Hatherley et al. 2015), Pubchem (Kim et al. 2016), ChemSpider (Pence and Williams 2010), and chEMBL (Gaulton et al. 2012) in form of PDB format. energy minimized using default Chimera parameters. Spike Domain PDB file downloaded from PDB (Berman et al. 2000) database ID: 6MOJ and separated from the ACE2 domain in a different file. while the $6 \mathrm{MOJ}$ is the complex of Spike domain bounded to the ACE2 domain. Spike extracted, minimized, and converted to PDBQT format in a separate file.

\section{Converting and Docking}

To loop and dock the 716 ligands, we build python script as shown in figure(1) to convert PDB ligand files to PDBQT using OpenBabel, export the converted file to Autodock Vina and docking against the Spike domain, and finally store each docked ligand with different orientation in the same file. All molecules docked against the whole Spike domain and the surface of Spike domain the containing the amino acids responsible for binding. Docking area dimensions which are center_x, center_y, center_z, size_x, size_y, and size_z for $-38.833,29.0981,-1.26252,32.9627,63.5716$, and 19.2879 respectively in the two processes determined using Chimera as shown in figure(2).

\section{Results And Discussion}

\section{Deep investigation}

we scanned the binding of the Spike domain with the ACE2 domain figure(3) to discover amino acid responsible for connecting the two domains figure(4-6). The investigation shows the main players in the binding process as shown in table(1). the table shows that Glycin is the most present amino acid in Spike and Glutamine in ACE2. 


\begin{tabular}{|ll|}
\hline In Spike & In ACE2 \\
\hline THR 500 & TYR 41 \\
\hline GLN 498 & GLN 42 \\
\hline GLY 446 & GLN 42 \\
\hline GLY 496 & LYS 353 \\
\hline ASN 501 & LYS 353 \\
\hline GLY 502 & LYS 353 \\
\hline TYR 505 & GLU 37 \\
\hline GLN 493 & GLU 35, HIS 34 \\
\hline ASN 487 & GLN 24, TYR 83 \\
\hline LYS 417 & ASP 30 \\
\hline TYR449 & ASP 38, GLN 42 \\
\hline
\end{tabular}

Table 1: list of amino acids responsible of binding to ACE2

\section{Docked NPs}

Molecular docking of 716 NPs reveals 12 candidate molecules to be used as Spike inhibitor with affinity $<=-8.0$ listed in the table(2) and shown in figure(7). 


\begin{tabular}{|c|c|c|c|}
\hline Name & Formula & 2D Structure & Affinity (kcal/mol) \\
\hline Cissacapine & $\mathrm{C}_{38} \mathrm{H}_{38} \mathrm{~N}_{2} \mathrm{O}_{6}$ & & -8.0 \\
\hline Bromotopsentin & $\mathrm{C}_{20} \mathrm{H}_{13} \mathrm{BrN}_{4} \mathrm{O}_{2}$ & & -8.2 \\
\hline Sodwanone R & $\mathrm{C}_{30} \mathrm{H}_{46} \mathrm{O}_{4}$ & & -8.0 \\
\hline P57 (glycoside) & $\mathrm{C}_{47} \mathrm{H}_{74} \mathrm{O}_{15}$ & & -8.3 \\
\hline Octahydroeuclein & $\mathrm{C}_{22} \mathrm{H}_{22} \mathrm{O}_{6}$ & & -8.3 \\
\hline 20(29)-Lupene-3 $\beta$-isoferulate & $\mathrm{C}_{40} \mathrm{H}_{58} \mathrm{O}_{4}$ & & -8.2 \\
\hline Sutherlandioside A & $\mathrm{C}_{36} \mathrm{H}_{60} \mathrm{O}_{10}$ & & -8.0 \\
\hline Scutiaquinone B & $\mathrm{C}_{32} \mathrm{H}_{30} \mathrm{O}_{6}$ & & -8.0 \\
\hline Sutherlandioside C & $\mathrm{C}_{36} \mathrm{H}_{58} \mathrm{O}_{10}$ & & -8.0 \\
\hline Sutherlandioside D & $\mathrm{C}_{36} \mathrm{H}_{58} \mathrm{O}_{9}$ & & -8.1 \\
\hline Saundersioside G & $\mathrm{C}_{46} \mathrm{H}_{66} \mathrm{O}_{15}$ & & -8.1 \\
\hline
\end{tabular}




\begin{tabular}{|l|l|l|l|} 
& & & \\
& & & \\
\hline Ornithosaponin A & $\mathrm{C}_{38} \mathrm{H}_{58} \mathrm{O}_{15}$ & & \\
& & & \\
\hline
\end{tabular}

Table 2: selected molecule and their binding affinity to Spike

\section{NPs-Spike Interaction}

By performing the docking procedure, it is clear that ligands can interact with amino acids as shown in figure (7) that generated by Chimera and Ligplot+ to show interacted amino acids using hydrogen and hydrophobic bonds between the Spike domain and selected molecules.

\section{Druggability Assessment}

After docking, we test the druggability of the 12 molecules using SwissADME (Daina, Michielin, and Zoete 2017) with is a web tool to determine drug-likeness and medicinal chemistry friendliness of each molecule as shown in figure (8). SwissADME also hosts the Brain Or Intestinal Estimated permeation method (BOILED-Egg) (Daina and Zoete 2016) which is a method proposed as an accurate predictive model that calculating the lipophilicity and polarity of small molecules to determine its permeation both brain and intestine as shown in figure (9) and the rest of analysis provided in supplementary(1).

\section{Target Prediction and Network Construction}

To identify the possible target macromolecule for each NPs we used SwissTargetPredict (Daina, Michielin, and Zoete 2019) give a probability for the query molecule - assumed as bioactive - to have this protein as a target. The analysis reveals that Bromotopsentin has a $100 \%$ probability to target for Alpha$1 \mathrm{~b}$ adrenergic receptors, and Octahydroeuclein has no targets. The rest of each molecule probabilities provided in supplementary(2). to build the network, We used SNPector's (Habib et al. 2020) Network construction python script to build and visualize a network show the unique and shared targets between different molecules and generate an HTML interactive network as shown in figure (10). we also built a network to show the unique and shared target classes, probabilities, and genes.

Having activity on alpha receptors in the human body predicts a spectrum of adverse effects, related mainly to the cardiovascular system, whereas Octahydroeuclein is expected to be safe. Octahydroeuclein was previously reported in the literature only in few publications. It was widely used for curing bronchitis, 
pleurisy, chronic asthma, and urinary tract infections as traditional medicine, by the Zulus, in South Africa. Also, octahydroeuclein was found to be significantly effective against Phytophthora (Lall et al. 2006), antifungal activity against A. niger, and Cladosporium cladosporioides respectively (Kothari et al. 2010; Aqil et al. 2010; Karthikeyan et al. 2020), and Antimycobacterial activity against Mycobacterium tuberculosis(Maroyi 2017). Docking against Spike revealed the best affinity with $-8.3 \mathrm{Kcal} / \mathrm{Mol}$ and best physicochemical proprieties which lead to recommending it as a possible drug for Coronavirus.

\section{Conclusion}

Novel Coronavirus represents a new formula of virulence and a real challenge to scientists all over the world. Natural products have shown their capacity to treat different diseases specially marine compounds. After docking 716 molecule, only 12 candidate molecules/compounds shows promising affinity in inhibiting or blocking Spike binding domain. in this study, we suggest Octahydroeuclein be one of the highest druggability and promising effects as a novel antiviral agent against SARS-CoV-2. Octahydroeuclein is photochemical has anti-fungal activity and shows potential to block Spike protein which may drive the efforts over the world to investigate and test with molecular docking of marine compounds against invulnerable viruses such as Coronavirus.

\section{Declarations}

Data Availability: Results and Scripts along with Supplementary Files 1 and 2 freely available on Github: zenodo.org/record/3888898\#.XuE6TnUzZnw

Competing interests: The authors have declared that no competing interests exist.

\section{References}

Adem, Sevki, Volkan Eyupoglu, Iqra Sarfraz, Azhar Rasul, and Muhammad Ali. 2020. "Identification of Potent COVID-19 Main Protease (Mpro) Inhibitors from Natural Polyphenols: An in Silico Strategy Unveils a Hope against CORONA."

Aqil, Farrukh, Maryam Zahin, lqbal Ahmad, Mohd Owais, Mohd Sajjad Ahmad Khan, Shyam S Bansal, and S Farooq. 2010. "Antifungal Activity of Medicinal Plant Extracts and Phytocompounds: A Review." In Combating Fungal Infections, 449-84. Springer.

Baron, Sophie Alexandra, Christian Devaux, Philippe Colson, Didier Raoult, and Jean-Marc Rolain. 2020. "Teicoplanin: An Alternative Drug for the Treatment of Coronavirus COVID-19." Int J Antimicrob Agents 105944 (10.1016). 
Berman, Helen M, John Westbrook, Zukang Feng, Gary Gilliland, Talapady N Bhat, Helge Weissig, llya N Shindyalov, and Philip E Bourne. 2000. "The Protein Data Bank." Nucleic Acids Research 28 (1): 235-42.

Bessong, Pascal Obong, Chikwelu Larry Obi, Marie-Line Andréola, Luis B Rojas, Laurent Pouységu, Eunice Igumbor, J J Marion Meyer, Stéphane Quideau, and Simon Litvak. 2005. "Evaluation of Selected South African Medicinal Plants for Inhibitory Properties against Human Immunodeficiency Virus Type 1 Reverse Transcriptase and Integrase." Journal of Ethnopharmacology 99 (1): 83-91.

Butler, Mark S, Avril A B Robertson, and Matthew A Cooper. 2014. "Natural Product and Natural Product Derived Drugs in Clinical Trials." Natural Product Reports 31 (11): 1612-61.

Clarkson, Cailean, Vinesh J Maharaj, Neil R Crouch, Olwen M Grace, Pamisha Pillay, Motlalepula G Matsabisa, Niresh Bhagwandin, Peter J Smith, and Peter I Folb. 2004. "In Vitro Antiplasmodial Activity of Medicinal Plants Native to or Naturalised in South Africa." Journal of Ethnopharmacology 92 (2-3): 17791.

Colson, Philippe, Jean-Marc Rolain, and Didier Raoult. 2020. "Chloroquine for the 2019 Novel Coronavirus SARS-CoV-2." International Journal of Antimicrobial Agents 55 (3): 105923.

Cortegiani, Andrea, Giulia Ingoglia, Mariachiara Ippolito, Antonino Giarratano, and Sharon Einav. 2020. "A Systematic Review on the Efficacy and Safety of Chloroquine for the Treatment of COVID-19." Journal of Critical Care.

Cragg, Gordon M, and David J Newman. 2013. "Natural Products: A Continuing Source of Novel Drug Leads." Biochimica et Biophysica Acta (BBA)-General Subjects 1830 (6): 3670-95.

Daina, Antoine, Olivier Michielin, and Vincent Zoete. 2017. "SwissADME: A Free Web Tool to Evaluate Pharmacokinetics, Drug-Likeness and Medicinal Chemistry Friendliness of Small Molecules." Scientific Reports 7: 42717.

---. 2019. "SwissTargetPrediction: Updated Data and New Features for Efficient Prediction of Protein Targets of Small Molecules." Nucleic Acids Research 47 (W1): W357--W364.

Daina, Antoine, and Vincent Zoete. 2016. "A Boiled-Egg to Predict Gastrointestinal Absorption and Brain Penetration of Small Molecules." ChemMedChem 11 (11): 1117.

Davies-Coleman, Michael T, and Denzil R Beukes. 2004. "Ten Years of Marine Natural Products Research at Rhodes University." South African Journal of Science 100 (11-12): 539-44.

DeLano, Warren L, and others. 2002. "Pymol: An Open-Source Molecular Graphics Tool.” CCP4 Newsletter on Protein Crystallography 40 (1): 82-92.

Dias, Daniel A, Sylvia Urban, and Ute Roessner. 2012. "A Historical Overview of Natural Products in Drug Discovery." Metabolites 2 (2): 303-36. 
Elfiky, Abdo A. 2020. "Ribavirin, Remdesivir, Sofosbuvir, Galidesivir, and Tenofovir against SARS-CoV-2 RNA Dependent RNA Polymerase (RdRp): A Molecular Docking Study." Life Sciences, 117592.

Elfiky, Abdo A, Samah M Mahdy, and Wael M Elshemey. 2017. "Quantitative Structure-Activity Relationship and Molecular Docking Revealed a Potency of Anti-Hepatitis C Virus Drugs against Human Corona Viruses." Journal of Medical Virology 89 (6): 1040-47.

Gao, J, and Z Tian. n.d. "Yang X. Breakthrough: Chloroquine Phosphate Has Shown Apparent Efficacy in Treatment of Covld-19 Associated Pneumonia in Clinical Studies. Biosci Trends. 2020 Epub Ahead of Print." Press. Doi 10.

Gaulton, Anna, Louisa J Bellis, A Patricia Bento, Jon Chambers, Mark Davies, Anne Hersey, Yvonne Light, et al. 2012. "ChEMBL: A Large-Scale Bioactivity Database for Drug Discovery." Nucleic Acids Research 40 (D1): D1100-D1107.

Gautret, Philippe, Jean-Christophe Lagier, Philippe Parola, Line Meddeb, Morgane Mailhe, Barbara Doudier, Johan Courjon, et al. 2020. "Hydroxychloroquine and Azithromycin as a Treatment of COVID-19: Results of an Open-Label Non-Randomized Clinical Trial." International Journal of Antimicrobial Agents, 105949.

Ghosh, Rajesh, Ayon Chakraborty, Ashis Biswas, and Snehasis Chowdhuri. 2020. "Evaluation of Green Tea Polyphenols as Novel Corona Virus (SARS CoV-2) Main Protease (Mpro) Inhibitors--an in Silico Docking and Molecular Dynamics Simulation Study." Journal of Biomolecular Structure and Dynamics, $1-13$.

Glaser, Keith B, and Alejandro M S Mayer. 2009. "A Renaissance in Marine Pharmacology: From Preclinical Curiosity to Clinical Reality." Biochemical Pharmacology 78 (5): 440-48.

Griffiths, Charles L, Tamara B Robinson, Louise Lange, and Angela Mead. 2010. "Marine Biodiversity in South Africa: An Evaluation of Current States of Knowledge." PloS One 5 (8): e12008.

Habib, Peter T, Alsamman M Alsamman, Sameh E Hassanein, Ghada A Shereif, and Aladdin Hamwieh. 2020. "SNPector: SNP Inspection Tool for Diagnosing Gene Pathogenicity and Drug Response in a Naked Sequence." F1000Research 8 (2133): 2133.

Haefner, Burkhard. 2003. "Drugs from the Deep: Marine Natural Products as Drug Candidates." Drug Discovery Today 8 (12): 536-44.

Hatherley, Rowan, David K Brown, Thommas M Musyoka, David L Penkler, Ngonidzashe Faya, Kevin A Lobb, and Özlem Tastan Bishop. 2015. "SANCDB: A South African Natural Compound Database." Journal of Cheminformatics 7 (1): 29.

Karthikeyan, Gnanasekeran, Mallappa Kumara Swamy, Madheshwar Rajha Viknesh, Rajendran Shurya, and Natesan Sudhakar. 2020. "Bioactive Phytocompounds to Fight Against Antimicrobial Resistance." In 
Plant-Derived Bioactives, 335-81. Springer.

Kim, Sunghwan, Paul A Thiessen, Evan E Bolton, Jie Chen, Gang Fu, Asta Gindulyte, Lianyi Han, et al. 2016. "PubChem Substance and Compound Databases." Nucleic Acids Research 44 (D1): D1202--D1213.

Kothari, Vijay, Aesha Shah, Shivani Gupta, Amruta Punjabi, and Abhishikha Ranka. 2010. "Revealing the Antimicrobial Potential of Plants." International Journal of Biosciences and Technology 3 (1): 1-20.

Lall, N, O Weiganand, A A Hussein, and J J M Meyer. 2006. "Antifungal Activity of Naphthoquinones and Triterpenes Isolated from the Root Bark of Euclea Natalensis." South African Journal of Botany 72 (4): 579-83.

Laskowski, Roman A, and Mark B Swindells. 2011. “LigPlot+: Multiple Ligand--Protein Interaction Diagrams for Drug Discovery." ACS Publications.

Maroyi, Alfred. 2017. "Review of Ethnomedicinal Uses, Phytochemistry and Pharmacological Properties of Euclea Natalensis A. DC." Molecules 22 (12): 2128.

Mishra, Bhuwan B, and Vinod K Tiwari. 2011. "Natural Products: An Evolving Role in Future Drug Discovery." European Journal of Medicinal Chemistry 46 (10): 4769-4807.

Newman, David J, and Gordon M Cragg. 2012. "Natural Products as Sources of New Drugs over the 30 Years from 1981 to 2010." Journal of Natural Products 75 (3): 311-35.

O’Boyle, Noel M, Michael Banck, Craig A James, Chris Morley, Tim Vandermeersch, and Geoffrey R Hutchison. 2011. "Open Babel: An Open Chemical Toolbox." Journal of Cheminformatics 3 (1): 33.

Pelay-Gimeno, Marta, Judit Tulla-Puche, and Fernando Albericio. 2013. "'Head-to-Side-Chain' Cyclodepsipeptides of Marine Origin." Marine Drugs 11 (5): 1693-1717.

Pence, Harry E, and Antony Williams. 2010. "ChemSpider: An Online Chemical Information Resource." ACS Publications.

Pettersen, Eric F, Thomas D Goddard, Conrad C Huang, Gregory S Couch, Daniel M Greenblatt, Elaine C Meng, and Thomas E Ferrin. 2004. "UCSF Chimera-a Visualization System for Exploratory Research and Analysis." Journal of Computational Chemistry 25 (13): 1605-12.

Sharma, Arun Dev, and Inderjeet Kaur. 2020. "Jensenone from Eucalyptus Essential Oil as a Potential Inhibitor of COVID 19 Corona Virus Infection." Research \& Reviews in Biotechnology \& Biosciences 7 (1): $59-66$.

Sharma, Arun Dev, and others. 2020. “Eucalyptol (1, 8 Cineole) from Eucalyptus Essential Oil a Potential Inhibitor of COVID 19 Corona Virus Infection by Molecular Docking Studies." 
Trott, Oleg, and Arthur J Olson. 2010. "AutoDock Vina: Improving the Speed and Accuracy of Docking with a New Scoring Function, Efficient Optimization, and Multithreading." Journal of Computational Chemistry 31 (2): 455-61.

Vuuren, S F Van. 2008. "Antimicrobial Activity of South African Medicinal Plants." Journal of Ethnopharmacology 119 (3): 462-72.

Walls, Alexandra C, Young-Jun Park, M Alejandra Tortorici, Abigail Wall, Andrew T McGuire, and David Veesler. 2020. "Structure, Function, and Antigenicity of the SARS-CoV-2 Spike Glycoprotein." Cell.

Wang, M, R Cao, L Zhang, and others. n.d. "Remdesivir and Chloroquine Effectively Inhibit the Recently Emerged Novel Coronavirus (2019 NCoV) in Vitro [Published Online February 4, 2020]." Cell Res. Doi 10.

\section{Figures}

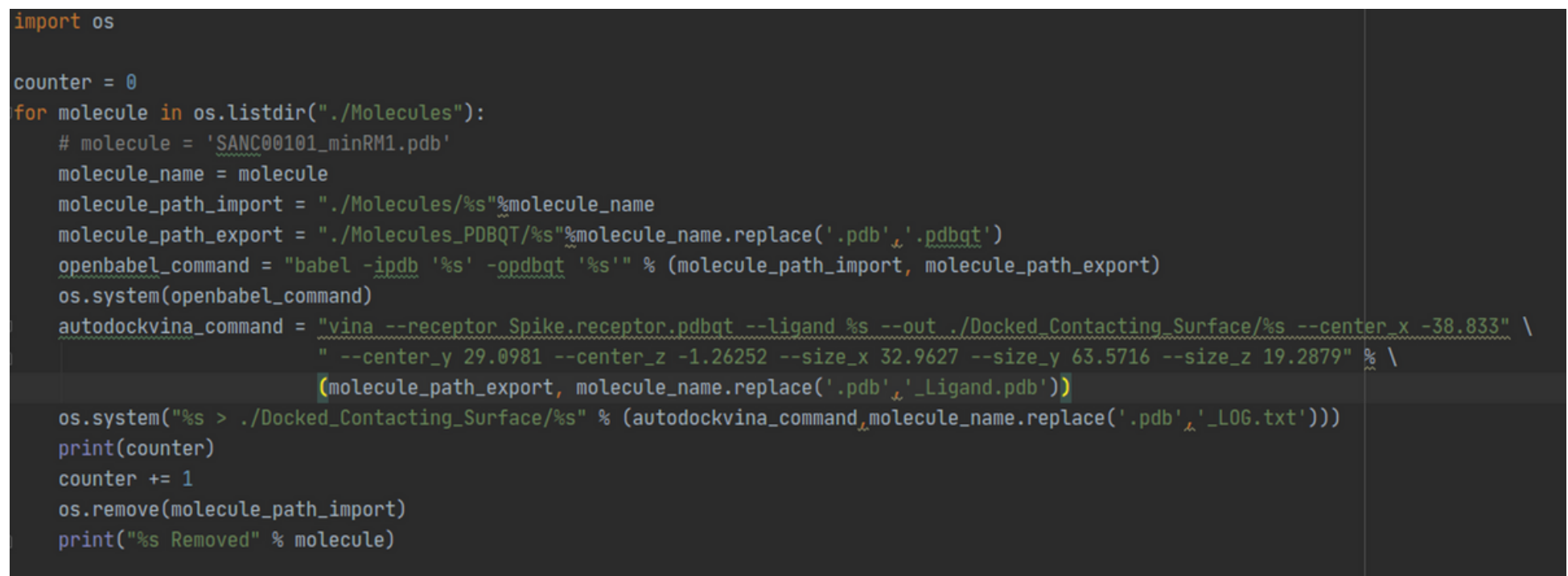

\section{Figure 1}

python script to convert and dock the molecules 


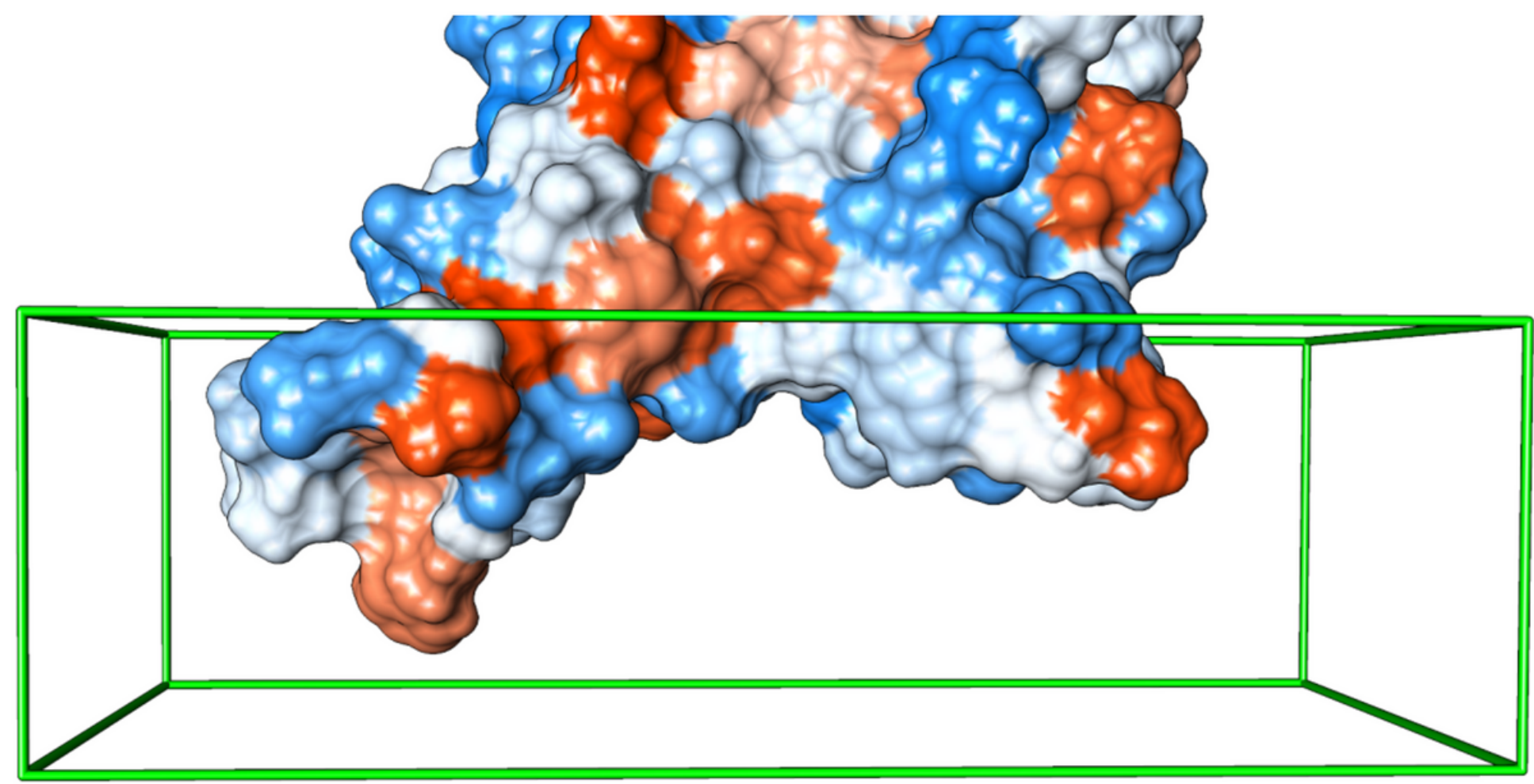

Figure 2

the docking area 


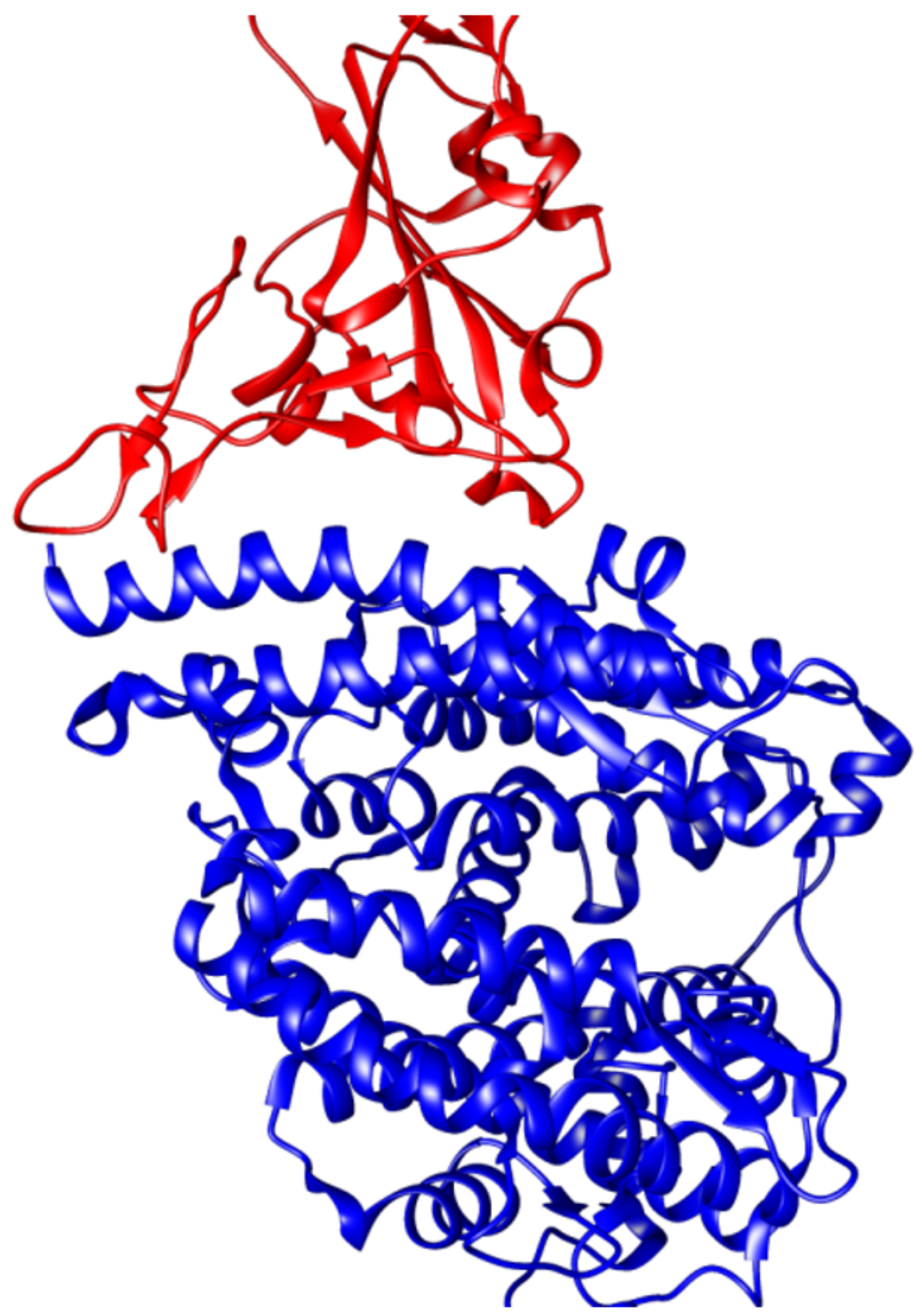

Figure 3

Spike domain (Red) with ACE2 domain (Blue) binding 


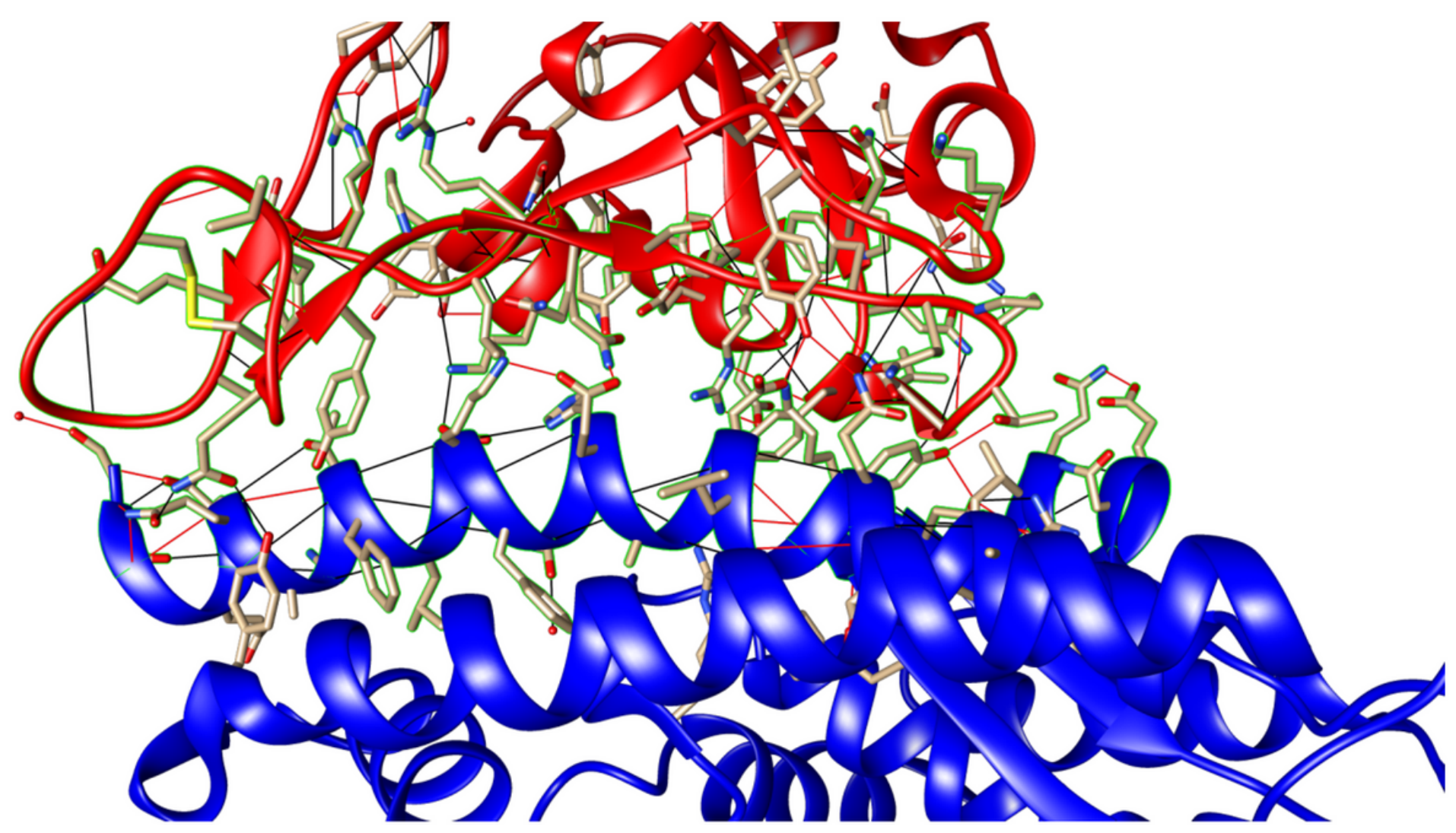

Figure 4

overview of interacting amino acids in both domains. Self and structural hydrogen bonds are in black, and the red bonds are bounding the two domains together.

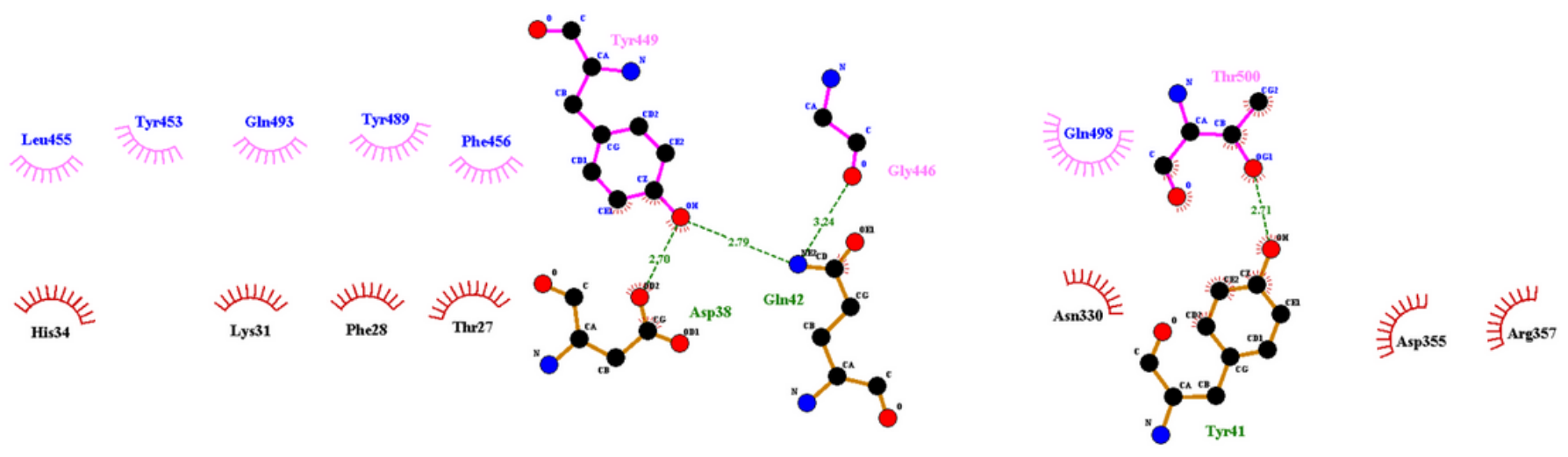

Figure 5

The Left Half of Spike-ACE2 interaction 

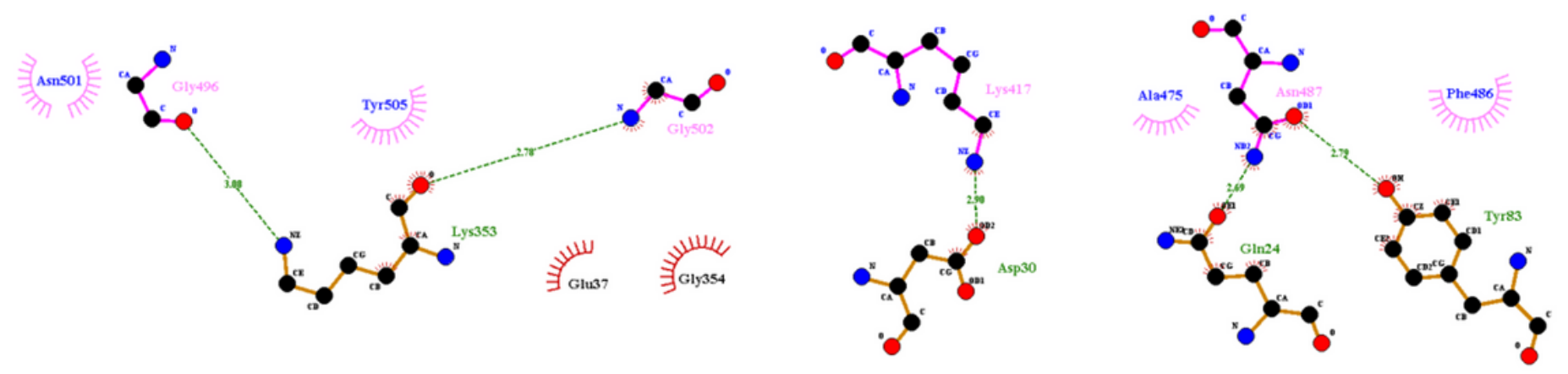

Figure 6

The Right Half of Spike-ACE2 interaction

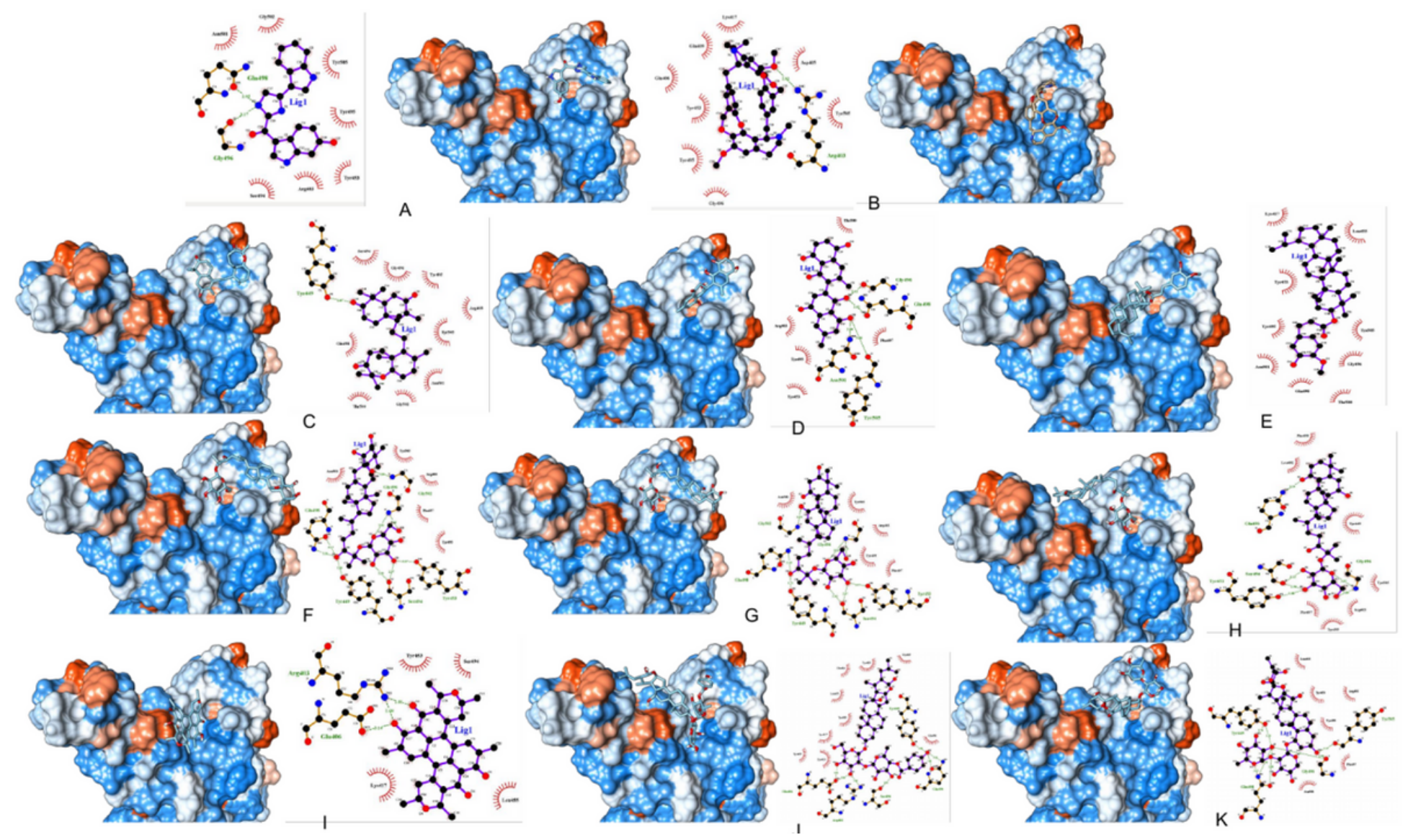

Figure 7

(A): 3D structure and 2D diagram of Spike-Cissacapine interaction, (B): 3D structure and 2D diagram of Spike-Bromotopsentin interaction, (C): 3D structure and 2D diagram of Spike-Sodwanone R interaction, (D): 3D structure and 2D diagram of Spike-Octahydroeuclein interaction, (E): 3D structure and 2D diagram of Spike-20(29)-Lupene-3 $\beta$-isoferulate interaction, (F): 3D structure and 2D diagram of SpikeSutherlandioside A interaction, (G): 3D structure and 2D diagram of Spike-Sutherlandioside C interaction, $(H)$ : 3D structure and 2D diagram of Spike-Sutherlandioside D Interaction, (I): 3D structure and 2D 
diagram of Spike-Scutiaquinone B Interaction, (J): 3D structure and 2D diagram of Spike-Saundersioside $\mathrm{G}$ Interaction, and (K): 3D structure and 2D diagram of Spike-Ornithosaponin A interaction.
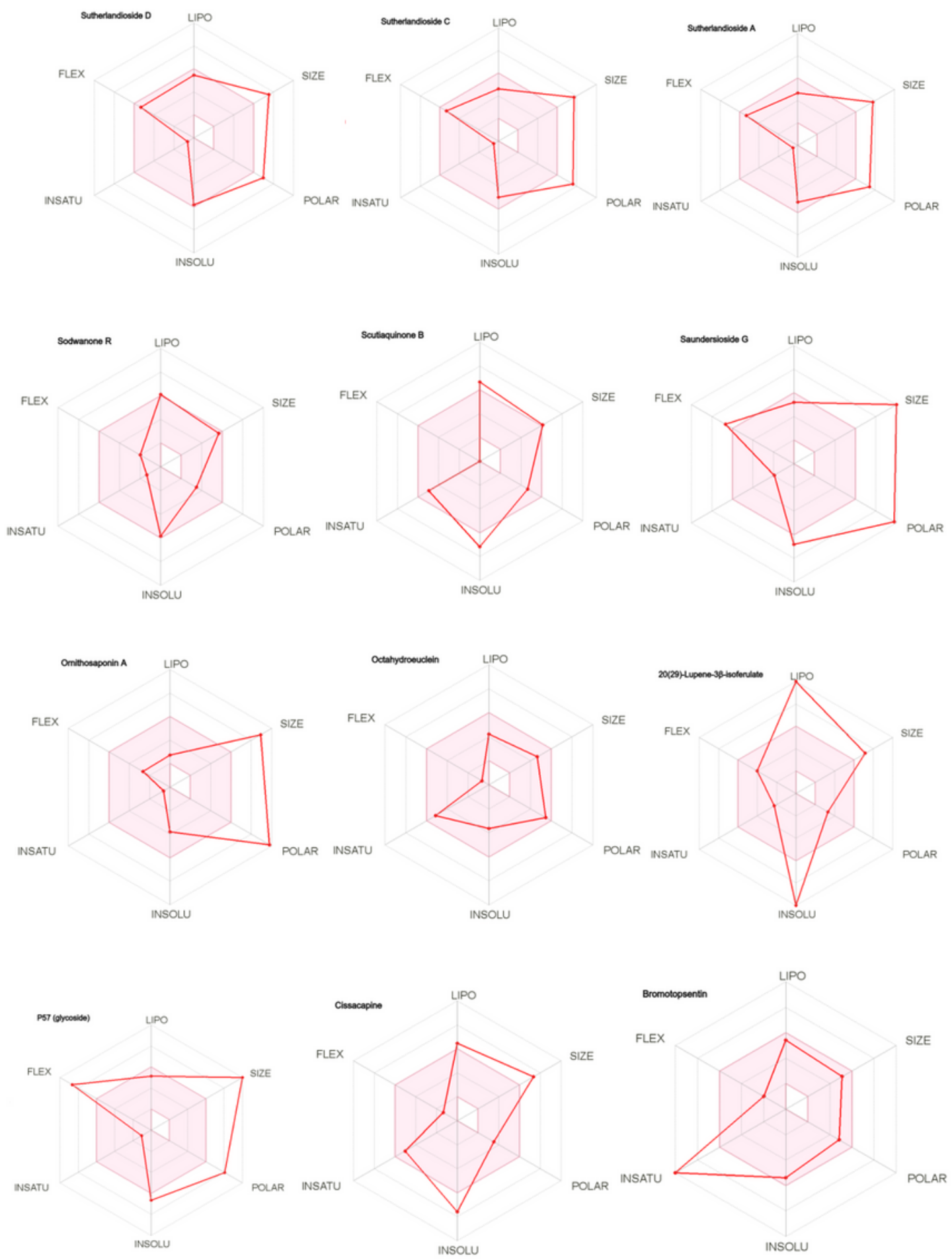

\section{Figure 8}

summary of SwissADME analysis where the colored zone is the suitable physicochemical space for oral bioavailability. LIPO: Lipophility, SIZE: Molecular Weight, POLAR: Polarity, INSOLU: Insolubility, INSTU: Insaturation, FLEX: Flexibility 
Sodwanone R

Scutiaquinone B

○

Cissacapine

$\stackrel{0}{\text { Bromotopsentin }}$

o

Sutherlandioside D

Octahydroeuclein suttetheaf

\section{Figure 9}

Points located in BOILED-Egg's yolk are molecules predicted to passively permeate through the bloodbrain barrier. Points located in the BOILED-Egg's white are molecules predicted to be passively absorbed by the gastrointestinal tract. Blue dots are for molecules predicted to be effluated from the central nervous system by the P-glycoprotein. Red dots are for molecules predicted not to be effluated from the central nervous system by the P-glycoprotein. 


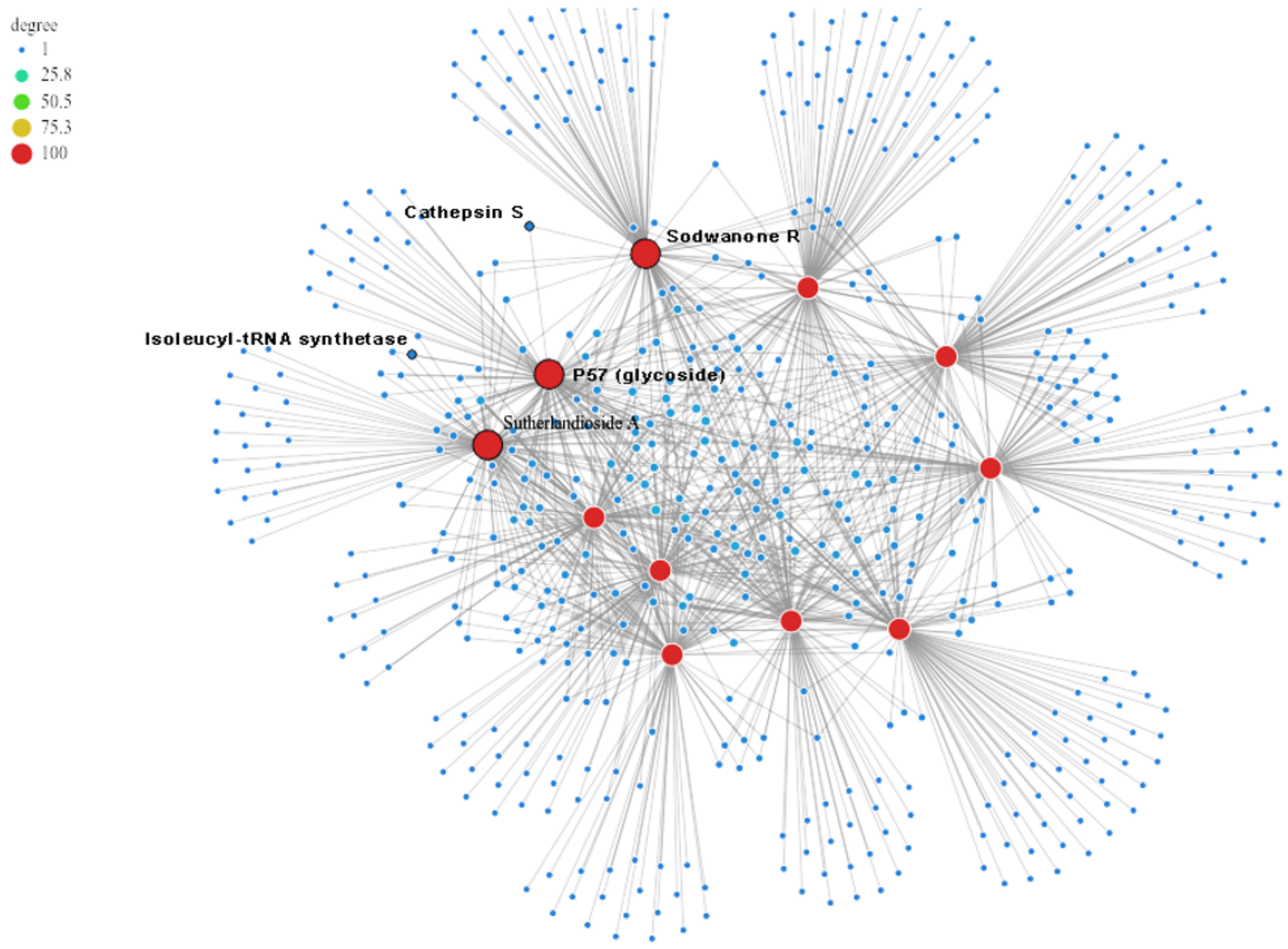

Figure 10

A network shows how different molecules have their unique target and also share targets with other molecules. Some molecule and targets highlighted for clarification. 